\title{
(4)
}

NASA Technical Memorandum 103153

ICOMP-90-14

\section{Metal Matrix Composites Microfracture: Computational Simulation}

Subodh K. Mital

Institute for Computational Mechanics in Propulsion

Lewis Research Center

Cleveland, Ohio

and

John J. Caruso and Christos C. Chamis

Lewis Research Center

Cleveland, Ohio

Prepared for the

Symposium on Computational Technology for Flight Vehicles cosponsored by NASA and George Washington University

Washington, D.C., November 5-7, 1990 


\title{
METAL MATRIX COMPOSITES MICROFRACTURE: COMPUTATIONAL SIMULATION
}

\author{
Subodh K. Mital* \\ Institute for Computational Mechanics in Propulsion \\ Lewis Research Center \\ Cleveland, Ohio 44135
}

and

\author{
John J. Caruso and Christos C. Chamis \\ National Aeconautics and Space Administration \\ Lewis Research Center \\ Cleveland, Ohio 44135
}

\section{ABSTRACT}

Fiber/matrix fracture and fiber-matrix interface debonding in a metal matrix composite (MMC) are computationally simulated. These simulations are part of a research activity to develop computational methods for microfracture, microfracture propagation and fracture toughness of the metal matrix composites. The three-dimensional finite element model used in the simulation consists of a group of nine unidirectional fibers in three by three unit cell array of SiC/Ti15 metal matrix composite with a fiber volume ratio of 0.35 . This computational procedure is used to predict the fracture process and establish the hierarchy of fracture modes based on strain energy release rate. It is also used to predict stress redistribution to surrounding matrix/fibers due to initial and progressive fracture of $\mathrm{fiber} / \mathrm{mat} \mathrm{ix}$ and due to debonding of fiber-matrix interface. Microfracture results for various loading cases such as longitudinal, transverse, shear and bending are presented and discussed. Step-by-step procedures are outlined to evaluate composite microfracture for a given composite system.

\section{INTRODUCT ION}

In a recent effort at NASA Lewis (ref. 1), three-dimensional finite element simulation was used to evaluate the effects of partial debonding on unidirectional composite (ply) properties. It was shown that, in general, single fiber fracture and/or debonding have little effect on most of the ply level properties. Among the material properties most effected are longitudinal modulus $E_{\ell 11}$ and longitudinal thermal expansion coefficient $\alpha \ell 11$ due to substantial fiber matrix debonding, particularly $\alpha_{211}$ making it a good indicator of the level of debonding in the composite. The present research is an extension of that work with emphasis on microfracture, fracture propagation and stress redistribution in surrounding matrix/fibers due to brittle fracture of fiber/ matrix or debonding of the fiber-matrix interface. The objective of this study is to develop a computational simulation procedure to predict the direction of crack propagation, based on strain energy release rate and to predict stress redistribution. Fiber/matrix fracture and fiber-matrix interface debonding has been simulated in the present work to predict fracture toughness. fracture propagation direction and extent of stress redistribution when the composite is subjected to longitudinal, transverse, shear or bending loads.

*Work funded by Space Act Agreement C-99066-G. 


\section{NOMENCLATURE}

$\begin{array}{ll}\text { d } & \text { diameter } \\ \text { E } & \text { Young's modulus } \\ \text { G } & \text { strain energy release rate } \\ \text { L } & \text { fiber length } \\ \mathrm{U} & \text { displacement in } X \text { direction } \\ v & \text { displacement in } Y \text { direction } \\ \text { w } & \text { displacement in } Z \text { direction } \\ \alpha & \text { coefficient of thermal expansion } \\ v & \text { Poisson's ratio }\end{array}$

\section{Subscripts:}

$\begin{array}{ll}\text { f } & \text { fiber } \\ \ell & \text { ply } \\ m & \text { matrix } \\ 11 & \text { is the longitudinal (along the fiber) direction } \\ 22,33 & \text { are transverse directions }\end{array}$

FINITE ELEMENT MODEL

The finite element model used in this computational simulation procedure consists of a group of nine fibers, all unidirectional, in a three by three unit cell array ("nine cell model") as shown in figure 1. The procedure is illustrated by using a composite system consisting of 35 percent fiber by volume SiC/Ti15 metal matrix composite with the properties as shown in table I. The finite element mesh consists of 16 "bays" along the length as shown in figure 2. Finite element mesh in each cell consists of $40 \mathrm{six}$-sided solid element with eight grid points (CHEXA) and 8 five-sided solid element with six grid points (CPENTA) elements. The overall mesh statistics are shown in figure 2 .

Various finite element analyses are made using MSC/NASTRAN version 65 (ref. 2). The load and boundary conditions are applied to the model through enforced displacements. The fiber diameter used is $223 \mu \mathrm{m}(8.8 \mathrm{mil})$. The length to diameter ratio of the $\mathrm{f} i b e r\left(\mathrm{~L} / \mathrm{d}_{\mathrm{f}}\right)$ is 6.8 and width of the interface is 6.8 percent of fiber diameter. In a typical set of simulations, fracture is initiated in the fiber at the middle of the center cell and is allowed to grow either in the matrix or along the fiber-matrix interface. Similarly, the crack could be initiated in the matrix or the fiber-matrix interface. Fracture is simulated by placing duplicate node points on either side of the crack. 
These duplicate nodal or grid points have the same geometrical location, but no connectivity exists between them, thus, in effect producing a crack of zero width. Cracks are introduced symetrically around the center fiber. For a given fracture configuration, fixed boundary displacements are applied to the model in a given direction. Resulting nodal forces corresponding to those applied displacements are found by the finite element analysis. Comparison of resulting nodal forces is made for reduction in global stiffness as the fracture propagates. The corresponding strain energy release rates are computed for perturbed fracture configurations by using the respective elementary definitions as described below.

\section{STRAIN ENERGY RELEASE RATE}

Strain energy release rate (SERR) is a very commonly used indicator of the fracture toughness of a material. Strain energy release rate gives a measure of the amount of energy required to propagate a defect in the laminate. Hence, one can make a direct comparison of damage tolerances between different microfracture configurations, materials and geometries. It provides the capability to identify particular fracture damage influences, such as geometry effects, material property dependence etc. This approach has been very effective in a previous investigation by Wilt et al. (ref, 3), where the strain energy release rates have been used to predict general delaminations in a composite laminate.

One of the methods used to calculate strain energy release rate is the crack closure method. In this method, nodal displacements and the corresponding nodal forces at the crack tip location, are used to determine the amount of work required to close the crack, which has been extended by an incremental amount. This is continued until the strain energy release rate increases very rapidly for equal increments of crack growth. The damage extent at which such a rapid increase in strain energy release rate would occur is critical damage, beyond which the damage becomes unstable and laminate fracture is imminent.

The above approach is a local level or a microfracture approach since the amount of energy produced by the local displacements and forces at the crack tip location are used to calculate the corresponding strain energy release rate. However, in the present research, a global approach has been used to calculate the strain energy release rate. In this approach, applied nodal displacements and the corresponding nodal forces obtained from finite element analysis at the loaded end of the composite are used to calculate the work done. Strain energy release rate is then, calculated as:

$$
G=\frac{d W}{d A}=\frac{1}{2} \cdot \frac{F_{2} \cdot u-F_{1} \cdot u}{\Delta A}
$$

where

$u \quad$ applied displacement at the loaded end of the model

$\Delta A \quad$ area of the new surfaces generated

$F_{1}, F_{2}$ forces at the end nodes before and after $\Delta A$ 
This equation is simply incremental change in work divided by the incremental change in new surface area that opens up. The applied displacements, $u$, remain constant but the resulting force required to maintain that displacement changes because of the reduction in stiffness of the composite as the fracture propagates.

\section{CASES STUDIED AND RESULTS}

The cases studied and the results obtained therefrom are described for the different loading cases below.

\section{Long i tudinal Loading}

For the case of longitudinal loading, the model is fixed in X-direction on the back face $(u=0.0)$ ( $f$ ig. 1) and on the front face, a fixed displacement is applied in the X-direction $(u=0.0012$ in. or $0.03 \mathrm{~mm})$. For this loading case, the center cell fiber is assumed to fracture first and then the crack is allowed to grow either into the matrix or along the fiber-matrix interface as shown schematically in figure 3 . Due to the initiation and propagation of fracture, stresses are redistributed in the surrounding matrix and the neighboring fibers. The longitudinal stress variation along the length of the specimen in matrix and also in neighboring fiber due to center fiber fracture is shown in figure 4. Shear stress in the interface also increases as a result of $f$ iber fracture. For example, in the case of fiber fracture only, the longitudinal stresses in surrounding matrix become twice of those in the reference (no fracture) case. Similarly, longitudinal stresses in neighboring fibers increase by 15 percent as compared to the reference case (fig. 4). It should be noted that constituent stresses are normalized by average ply level stresses, which are obtained by summing up all the forces on the loaded face and dividing that by the surface area of that face. It is also observed that stresses redistribute around a microfracture within a short distance away from it and the stress concentration factors are lower than what would be expected in an isotropic case.

The reduction in global longitudinal stiffness as more fibers are fractured in a plane is shown in figure 5 . The reduction in global stiffness when all the fibers are fractured in a plane is only about 9 percent for this composite system. Hence, when all the fibers are fractured in one plane, there is a reduction in strength in that plane, but the reduction in global stiffness is small. It is well within the experimental scatter and perhaps difficult to detect experimentally (unless the strain gage bridges the fracture plane). The strain energy release rate as fibers are fractured in a plane is shown in figure 6. It shows that once, one out of nine (11 percent) fiber is fractured in the middle, it is the onset of instability. The crack, in this particular fracture mode, propagates without requiring any additional energy. This level of energy can also be thought of as the equivalent of fracture toughness of the material, which is, by definition, the level of energy at which crack propagates without requiring any additional energy. It also indicates that once one out of nine fibers is fractured, all other fibers can fracture at the same energy level, which will lead to a brittle failure. But, for such brittle fracture to happen, the interface has to be very strong, as will be discussed later. 
If only the center fiber is fractured at middle and the crack is allowed to grow along the $\mathrm{f}$ iber-matrix interface, then there is about 10 percent reduction in longitudinal stiffness for fully debonded center fiber, same as was reported in a previous study (ref. 4). There is a reduction in st iffness as the center fiber debonds following the fiber fracture as shown in $f$ igure 7 . The corresponding strain energy release rate versus the fiber length debonded is shown in figure $8(\mathrm{a})$. If the fracture initiates in the matrix and the crack is allowed to grow along the fiber-matrix interface without a fiber fracture, the reduction in stiffness is very small and thus, the strain energy release rates are also very small as shown in figure $8(\mathrm{~b})$. If the fracture initiates and progresses along the fiber-matrix interface, there is no reduction in the global longitudinal stiffness and hence the corresponding strain energy release rates are also zero. Thus, even though, it takes less energy to drive the crack in this fracture mode (debonding along fiber-matrix interface), one could not reach this state prior to fiber or matrix fracture (fig. 8(a)). In other words, the debonding will not take place by itself, it follows the fiber fracture. Figure 8(a) and (b) show similar type of behavior. If the matrix were to fracture first, interface debonding will follow instantaneously. Although, the energy required to propagate the crack in interface debonding mode following the matrix fracture is much less than the energy required when the debonding follows the fiber fracture. Also, from figures $8(a)$ and (b), it can be concluded that if the matrix strength is more than $1 / 20$ of the fiber strength, the fracture will not initiate in the matrix. Fracture will, then, initiate in the fiber and interface debonding will follow instantaneously. On the other hand, in the case of ceramic composites, for example, where the fiber and matrix stiffnesses are almost the same, but the matrix strength is much less than the fiber strength, then the fracture will initiate in matrix, followed by instantaneous interface debonding.

Hence, for the case of longitudinal loading, due to fracture in one fiber, the increase in longitudinal stress, $\sigma_{\ell 11}$, in neighboring fibers is small, implying that the random fracture in a fiber is unlikely to initiate fracture in neighboring fibers. Following a fiber fracture, depending upon the local matrix tensile strength and the local interface shear strength, the crack grows either in the matrix or along the fiber-matrix interface. It is also concluded that for a unidirectional composite subjected to longitudinal loading, interface debonding does not initiate by itself. It propagates along the fibermatrix interface as a followup of fiber or matrix fracture.

\section{Transverse Loading}

For the case of transverse loading, the bottom XY-plane (fig. 1) is fixed in Z-direction $(w=0.0)$ and the top XY-plane is given a fixed displacement in $\mathrm{Z}$-direction $(w=0.0008$ in. or $0.02 \mathrm{~mm})$. For this loading case, if the fracture initiates in the fiber or in the matrix, there is no reduction in the transverse stiffness, unlike the case of longitudinal loading when fiber fracture did cause a reduction in longitudinal stiffness. However, if the crack initiates at the fiber-matrix interface and as the fiber surface debonds from the matrix, there is a considerable reduction in transverse stiffness as the debonded fiber surface area increases. The reduction in transverse st iffness versus the percentage of fiber surface area debonded is shown in figure 9 . There is approximately 20 to 25 percent reduction in transverse stiffness when 50 percent of total fiber surface area is debonded. The corresponding strain 
energy release rate curve versus the percentage of fiber surface area debonded is shown in figure 10. The crack growth in this mode is stable. Once, 10 percent of total fiber surface area is debonded, it takes much less energy to drive the crack further, indicating crack propagation instability and high sensitivity of debonding extension due to transverse loading. Hence, it can be concluded that the transverse tensile test is much more indicative of interfacial conditions than it is of either fiber or matrix conditions.

\section{Shear Loading}

Shear loading is applied to the specimen both in XY-plane and YZ-plane. For the shear loading in the XY-plane, entire model is $f$ ixed in $Y$ and $Z$ direction (fig. 1). The left $X Z$-plane is fixed in X-direction ( $u=0.0)$ ( $f$ ig. 1) and right XZ-plane is given a prescribed displacement in the X-direction $(\mathrm{u}=0.0008 \mathrm{in}$. or $0.02 \mathrm{~mm})$. For the shear loading in the YZ-plane, entire model is fixed in the $X$ and $Y$ directions. The lef $t X Z$-plane is $f$ ixed in Z-direction and right XZ-plane is given a prescribed displacement in the Z-direction $(w=0.0008$ in. or $0.02 \mathrm{~mm})$. Results for both of these shear loadings are similar. If only a fiber or the matrix is fractured, it does not reduce the global shear stiffness of the composite, and hence, the strain energy release rates are negligibly small. However, there is a gradual decrease in shear stiffness as the center fiber starts to debond (fig. 11). There is approximately 6 percent reduction in stiffness as the center fiber is fully debonded. The corresponding strain energy release rate as the center fiber debonds is shown in figure 12. The fracture propagation in this loading case is stable. Additional energy needed to drive the crack reduces as the fracture propagates along the fiber-matrix interface. Thus, the shear loading shows the same type of behavior as was observed under transverse loading i.e., shear loading is much more indicative of the interfacial conditions than it is of either fiber or matrix conditions.

\section{Bending Load}

Load was applied on the model so as to bend it in the XZ-plane (refer to fig. 1). The model was fixed in the $z$-direction $(w=0.0)$ on the back face $(\mathrm{X} / \mathrm{L}=0$, fig. 1). On the front face $(\mathrm{X} / \mathrm{L}=1)$, a total vertical load of $445 \mathrm{~N}$ $(100 \mathrm{lb})$ was applied in the - $z$ direction equally distributed over the nine fiber surfaces (fig. 13). Resulting displacements corresponding to the applied nodal forces are found by finite element analyses for different fracture configurations. In one set of simulations, one fiber in top layer was fractured at the middle and then all three fibers were fractured. Similarly, the crack was initiated in the matrix and in all cases it was allowed to propagate along the fiber-matrix interface following the fiber/matrix fracture. It was found that there is no reduction in global bending stiffness when fiber/matrix fracture or fiber-matrix interface debonds.

Even though, the fiber or matrix fracture has no effect on the global bending stiffness, it provides a site for the initiation of delamination. Hence, the effect of delamination on the global bending stiffness was also investigated. The delamination was initiated between the top and middle plies symmetrically about the middle plane $(X / L=0.5)$. The delamination is then propagated outward towards the free edges of the laminate and also along the 
length. It is found that the internal (interior) delaminations (fig. 14) do not reduce the global stiffness in bending. Only, when the delamination extends over the full width of the laminate, there is approximately 10 percent reduction in global bending stiffness as compared to the case with no delamination. The reduction in global bending stiffness is independent of the extent of delamination longitudinally even when it extends over the full width. The strain energy release rate versus percentage of area delaminated curve is shown in figure 15. It shows that once interior delamination, extending up to full width initiates, it is the onset of instability. The delamination can extend longitudinally at the same fracture energy level. The reason being that the shear which is driving the delamination is constant along the length.

\section{PROCEDURES FOR EVALUATING COMPOSITE MICROFRACTURE}

The collective results from the computational simulations previously described can be used to provide step-by-step procedures for evaluating composite microfracture. It can be used to predict fracture toughness, fracture process, hierarchy of fracture modes and extent of stress redistribution in a given composite system for various loading cases. The following steps outline the procedure for evaluating composite microfracture:

(1) Obtain the room temperature properties of the fiber and matrix (constituent) materials.

(2) Generate a three-dimensional finite element model as described in the Finite Element Model section.

(3) Perform the simulation for the reference case (no fracture) under desired loading condition (longitudinal, transverse etc.).

(4) Initiate the fracture in the fiber and perform the simulation under same loading condition as in step 3 . Then, propagate the fracture in the matrix and/or along the interface and perform various computational simulations for different extent of damage.

(5) Repeat step 4, except that initiate the fracture in the matrix or in the fiber-matrix interface.

(6) For a given loading condition, compare the different fracture configurations for reduction in global stiffness and corresponding strain energy release rates.

(7) Determine the most likely direction for crack propagation for a given loading condition based on the strain energy release rates. It may happen that a particular fracture configuration requires a very small amount of $f$ racture energy to drive the crack, but a certain other fracture configuration requiring high fracture energy must be reached prior to reaching a fracture configuration requiring a low fracture energy (fig. $8(\mathrm{a}))$.

(8) Knowing the fiber, matrix and interface in-situ strengths and strain energy release rates for various loading conditions and fracture configurations, estimate the most likely direction of fracture propagation as well as fracture modes and fracture sequence. 


\section{SUMMARY}

A three-dimensional finite element computational simulation procedure was developed for microfracture propagation and to evaluate fracture toughness in metal matrix composites based on strain energy release rates. The procedure was applied to simulate microfracture for fiber, matrix and fiber-matrix interface. Step-by-step procedures were outlined to predict the fracture process and establish hierarchy of fracture modes. The significant results from this work are as follows:

1. Fracture in one fiber is unlikely to initiate fracture in neighboring fibers when the composite is subjected to longitudinal loading. Also, the interface debonding will not initiate by itself under such loading. It will progress along the interface as a follow up of $f$ iber or matrix fracture.

2. Even if a substantial percentage of fibers are fractured in a plane, there is a reduction in the strength in that plane, but the reduction in global longitudinal stiffness is small and perhaps difficult to detect by experiments, at least for the composite system and fiber volume ratio investigated.

3. Debonding along the fiber-matrix interface is the only likely event for the fracture propagation for composites subjected to transverse or shear type loading. Although, under shear loading, the composite is not as sensitive to debonding extension as it is under transverse loading.

4. Composites subjected to bending loads show that the fiber/matrix frac ture or fiber-matrix interface debonding is not sensitive to bending loads. Delamination is the only likely mode of fracture propagation under such loading.

5. The stresses redistribute around a microfracture within a short distance for the composite system investigated. Also, the stress concentrations around a microfracture are lower than what would be expected for an isotropic case due to the composite being subjected to far field stresses.

\section{REFERENCES}

1. Caruso, J.J.; Trowbridge, D.; and Chamis, C.C.: Finite Element Applications to Explore the Effects of Partial Bonding on Metal Matrix Composite Properties. AIAA Paper 89-1175, Apr. 1989.

2. MSC/NASTRAN, Version 64, Vol. I and II, Users Manual, The MacNealSchwendler Corporation, Los Angeles, 1982.

3. Wilt, T.E.; Murthy, P.L.N.; and Chamis, C.C.: Fracture Toughness Computational Simulation of General Delamination in Fiber Composites. J. Reinf. Plast. and Compos.. vol. 8, no. 1, Jan. 1989, pp. 2-17.

4. Caruso, J.J.; and Chamis, C.C.: Superelement Vethods Applications to Micromechanics of High Temperature Metal Matrix Composites. Structures, Structural Dynamics and Materials Conference, 29th, Williamsburg, VA, Apr. 1988, Technical Papers, Pt. 3, AIAA, Washington, DC, 1988, pp. 1388-1400. (Also AIAA Paper 88-2390, 1988.) 
TABLE I . - PROPERTIES OF CONSTITUENT MATERIALS OF SiC/Ti15

\begin{tabular}{|l|c|c|c|}
\hline & SiC fiber & Ti15 matrix & Interface \\
\hline Modulus, E, mpsi & 62.0 & 12.3 & 12.3 \\
Poisson's ratio, $v$ & .3 & .32 & .32 \\
Shear mosulus, G, mpsi & 23.8 & 4.6 & 4.6 \\
Coef icient of thermal & 1.8 & 4.5 & 4.5 \\
expansion $\alpha, \mathrm{ppm} /{ }^{\circ} \mathrm{F}$ & & & \\
\hline
\end{tabular}

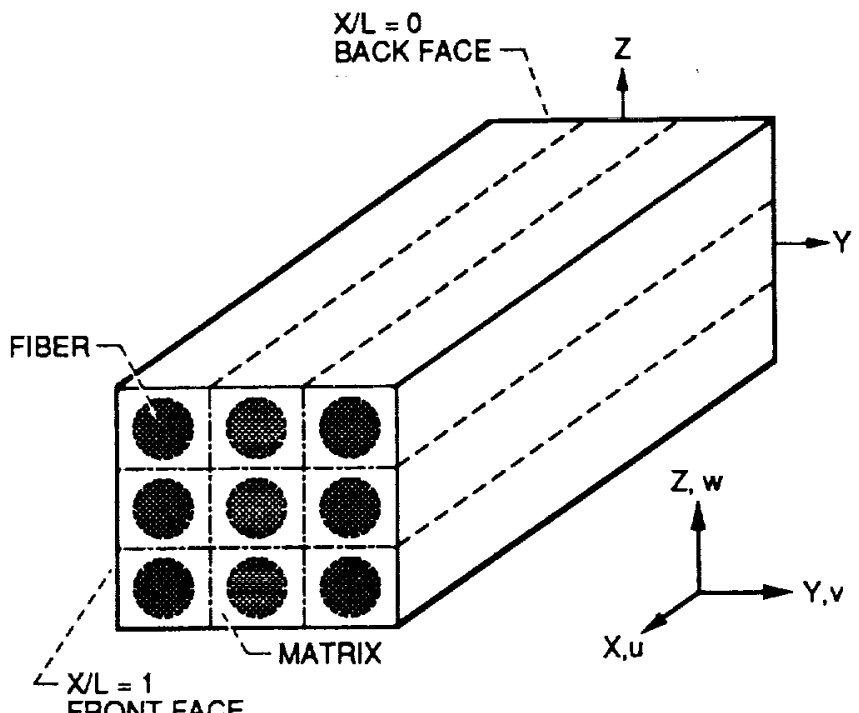

FRONT FACE

Figure 1. - Schematic diagram of nine cell model.

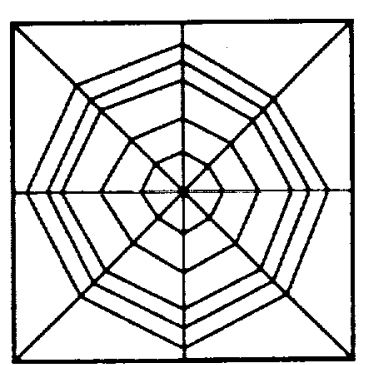

UNIT CELL

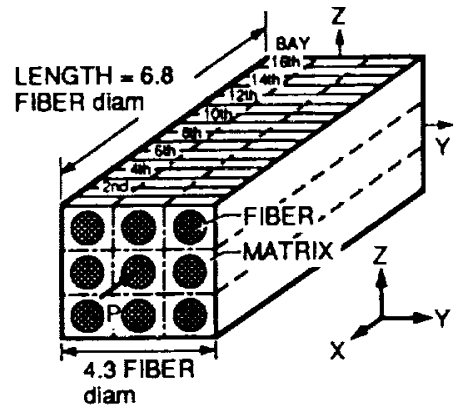

REPRESENTATION OF NINE CELL MODEL

TOTAL 6912 ELEMENTS 6953 NODES

Figure 2. - Representation of nine cell model finite element mesh.

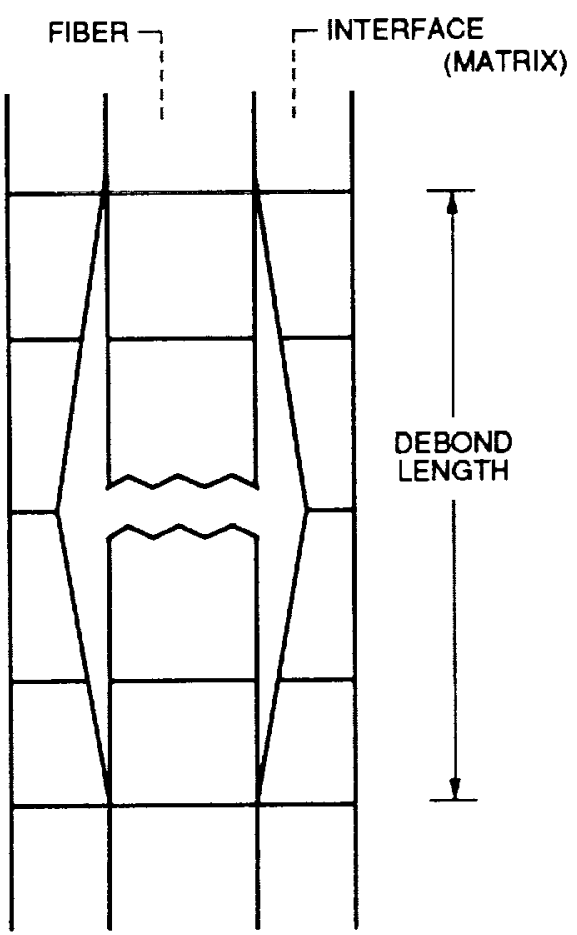

Figure 3. - Representation of fiber fracture and interface debonding. 

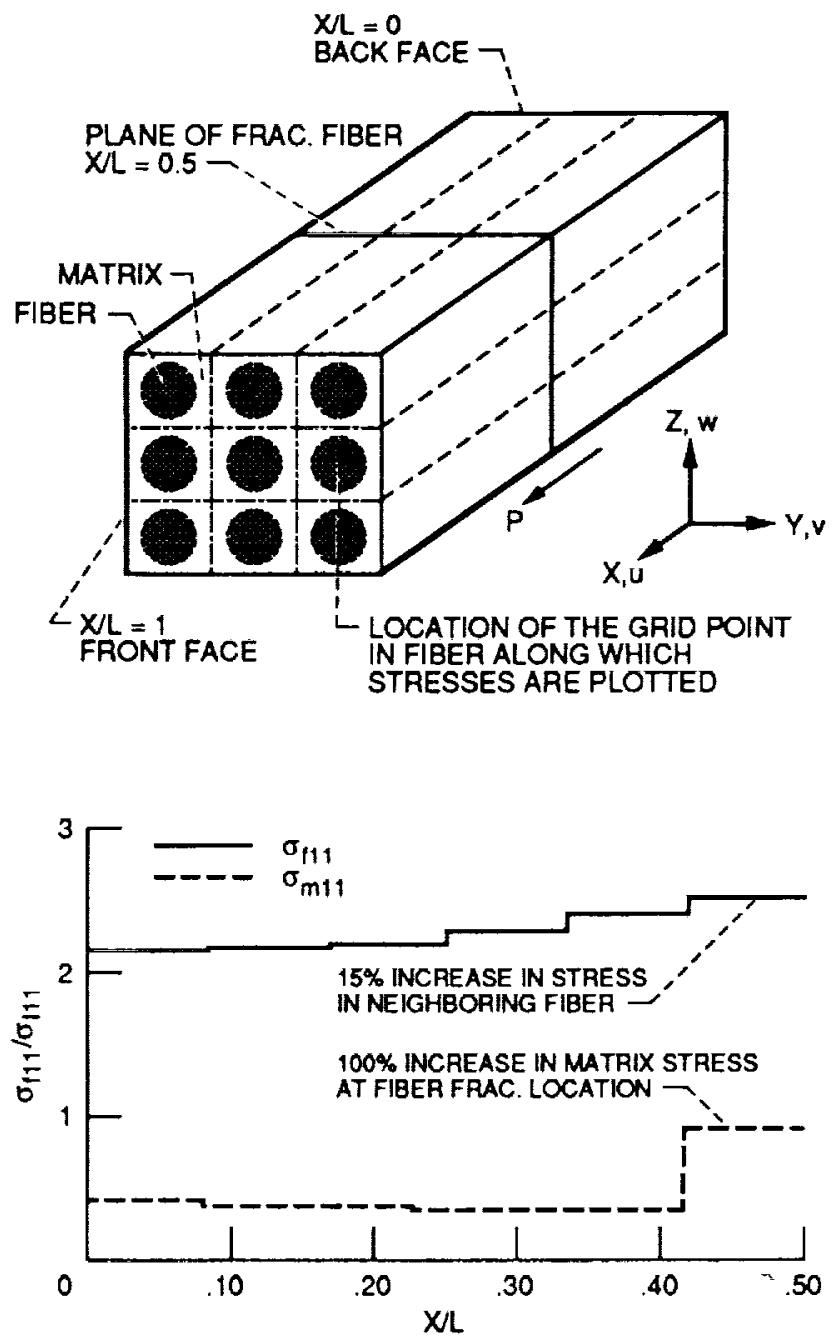

Figure 4. - Stress variation along the length in fiber and matrix for longitudinal loading.

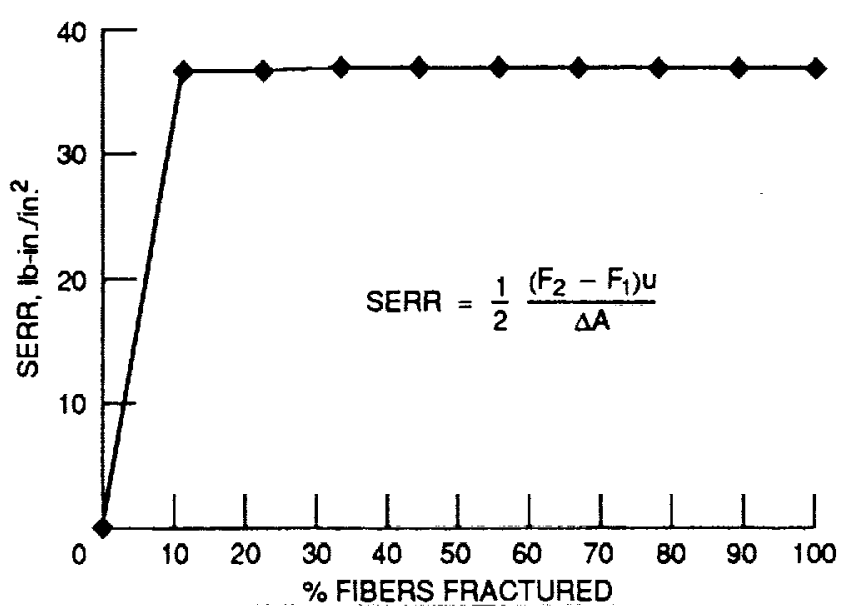

Figure 6. - Strain energy release rate vs. percent fibers fractured for longitudinal load.
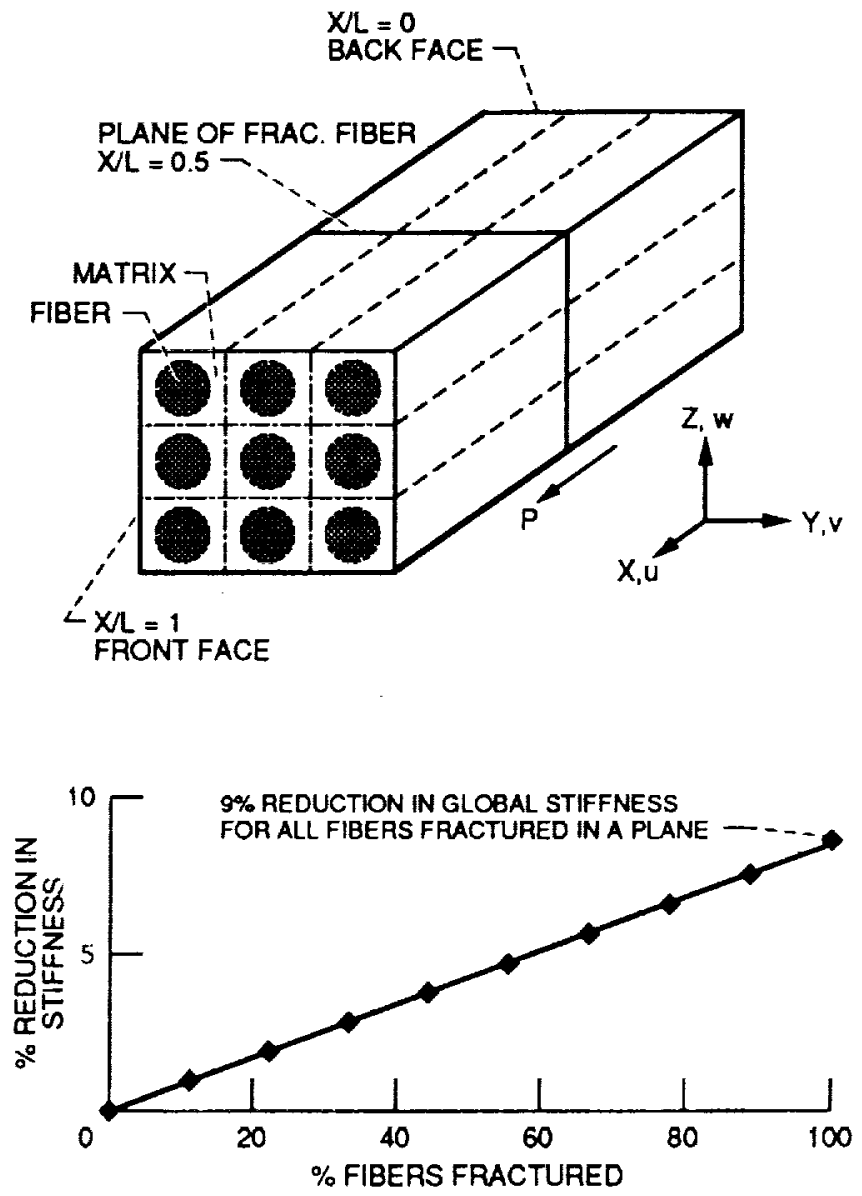

Figure 5. - Reduction in stiffness vs. percent fibers fractured for longitudinal loading.

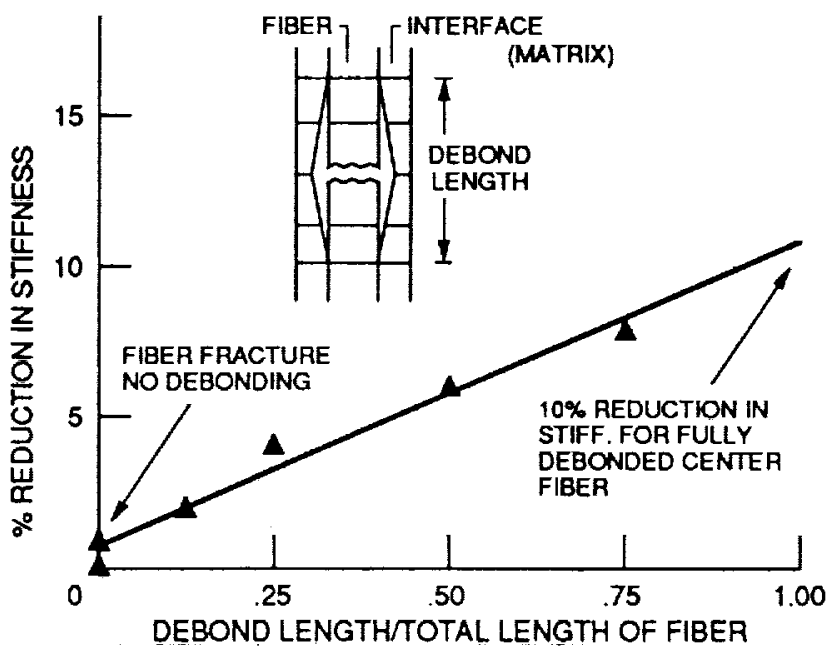

Figure 7. - Reduction in stiffness vs. debond length of center fiber for longiludinal loading. 

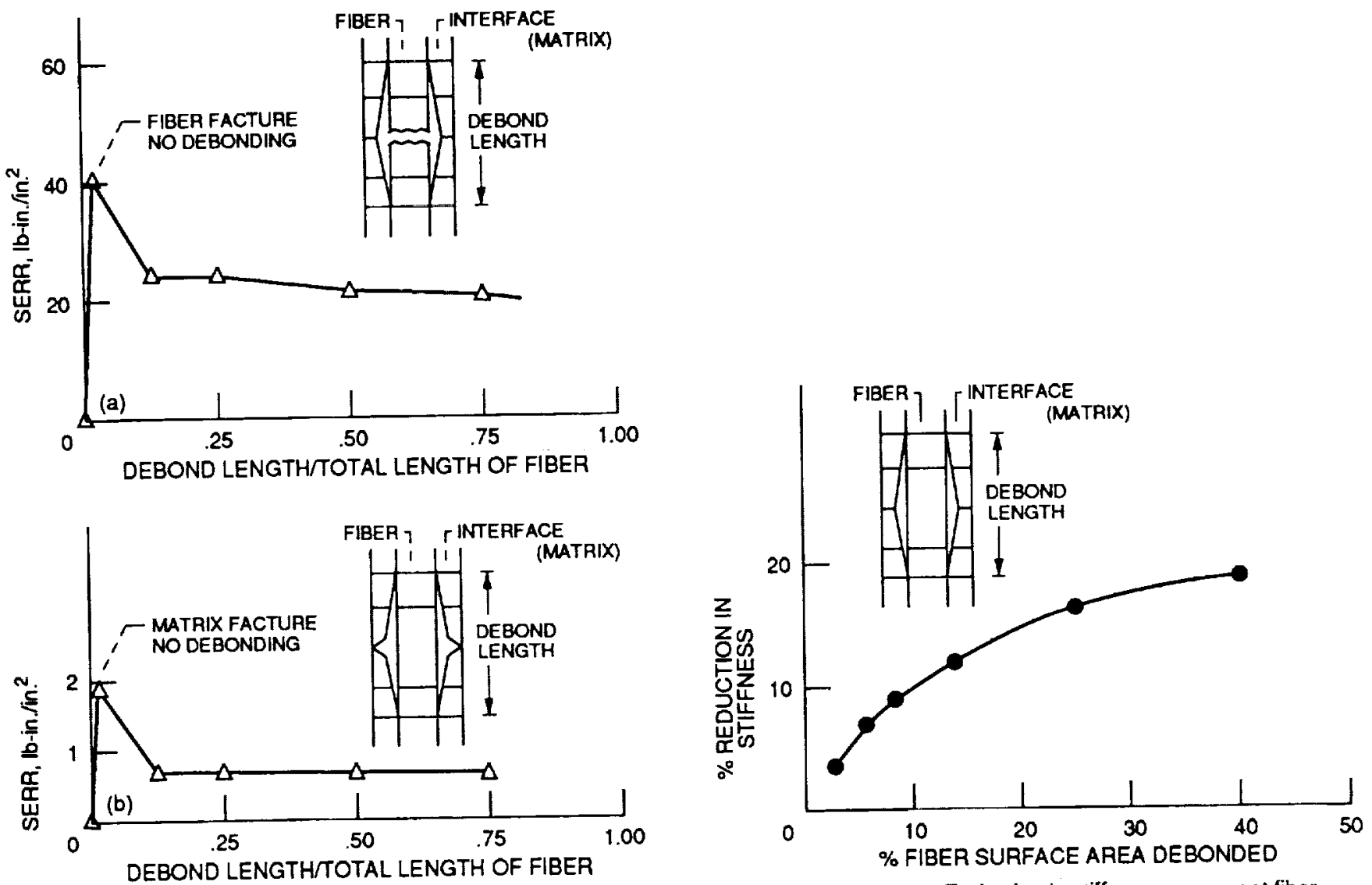

Figure 8. - Strain energy release rate for center fiber debonding for longitudinal loading.

Figure 9. - Reduction in stiffness vs. percent fiber surface area debonded for transverse loading.
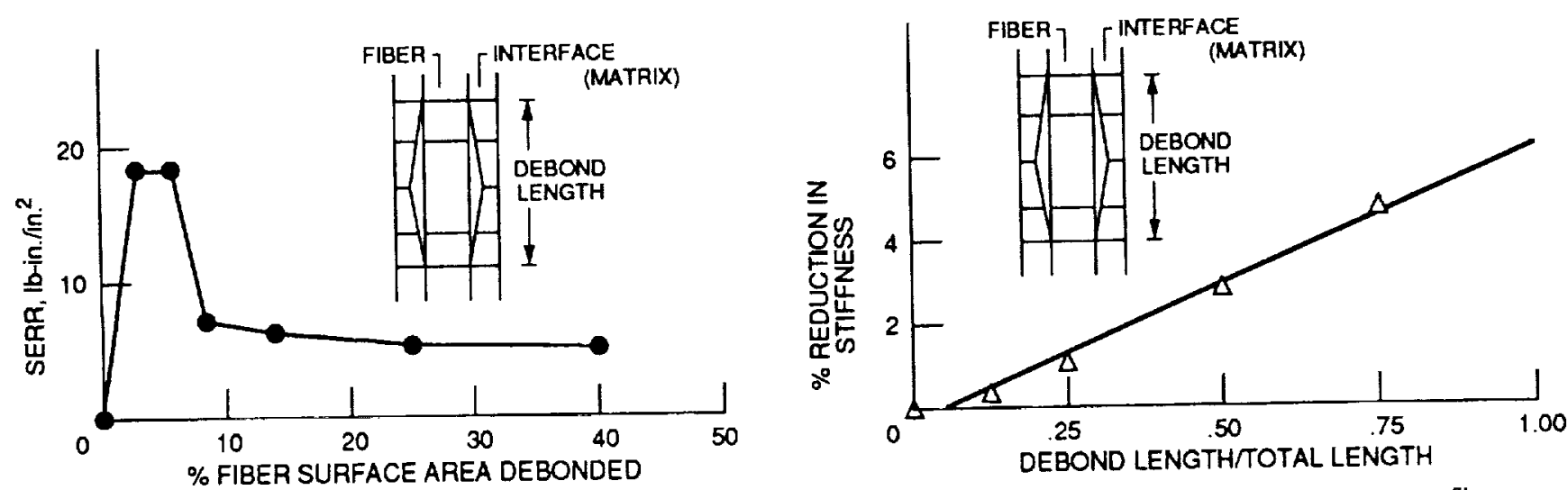

Figure 10. - Strain energy release rate vs. percent fiber surface area debonded for transverse loading.

Figure 11. - Reduction in stiffness vs. center fiber debonding lor $X Y$ shear loading. 


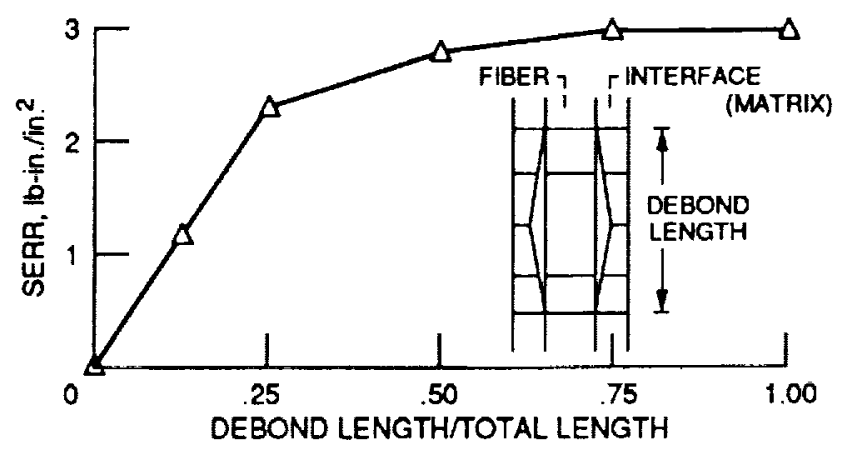

Figure 12. - Strain energy release rate for center fiber debonding for $X Y$ shear loading.

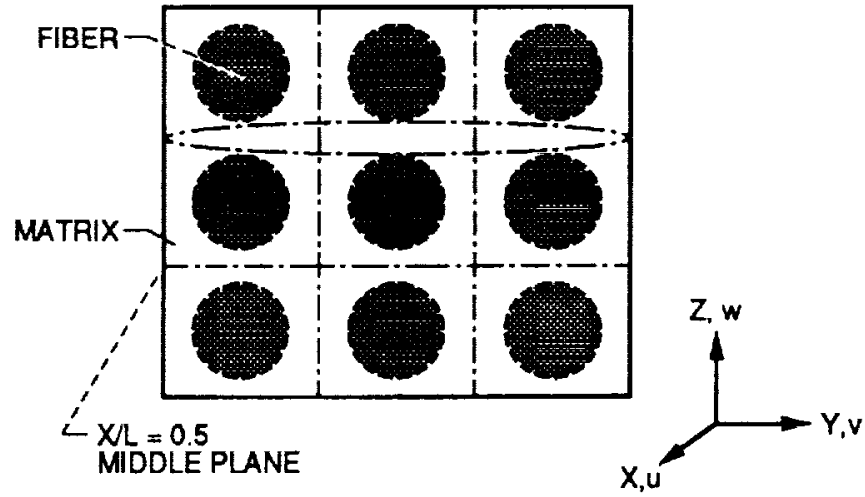

Figure 14. - Internal delamination between top and middle plies.

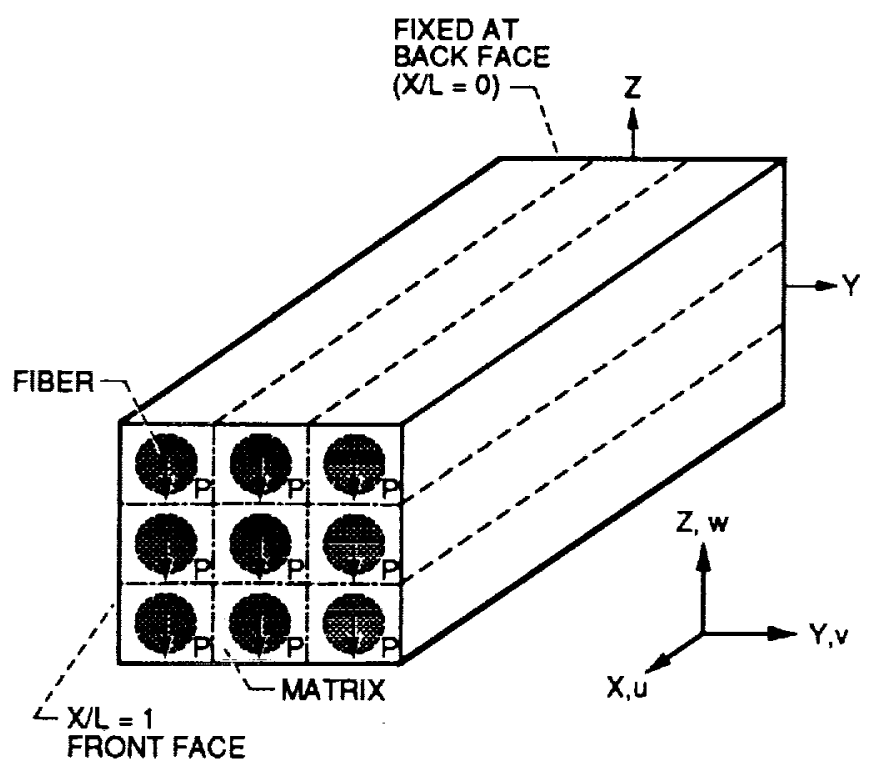

Figure 13. - Application of bending loads.

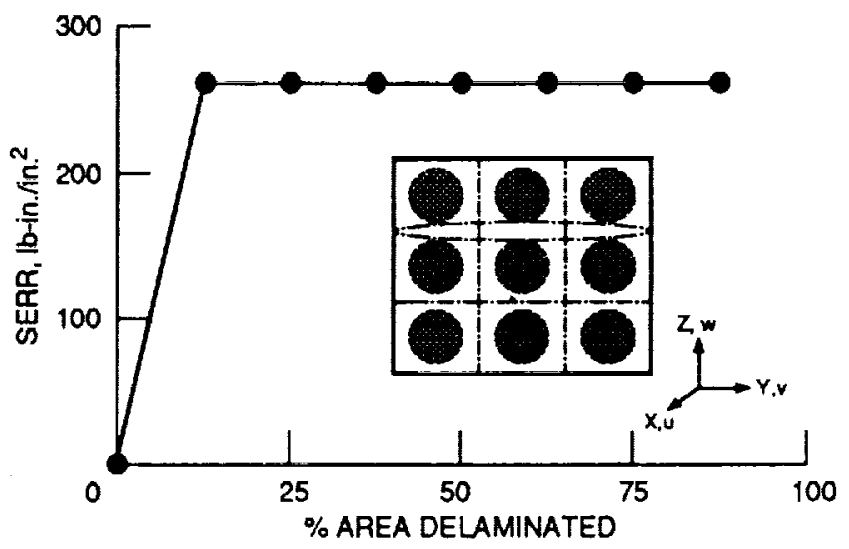

Figure 15. - Strain energy release rate for interior delamination. 


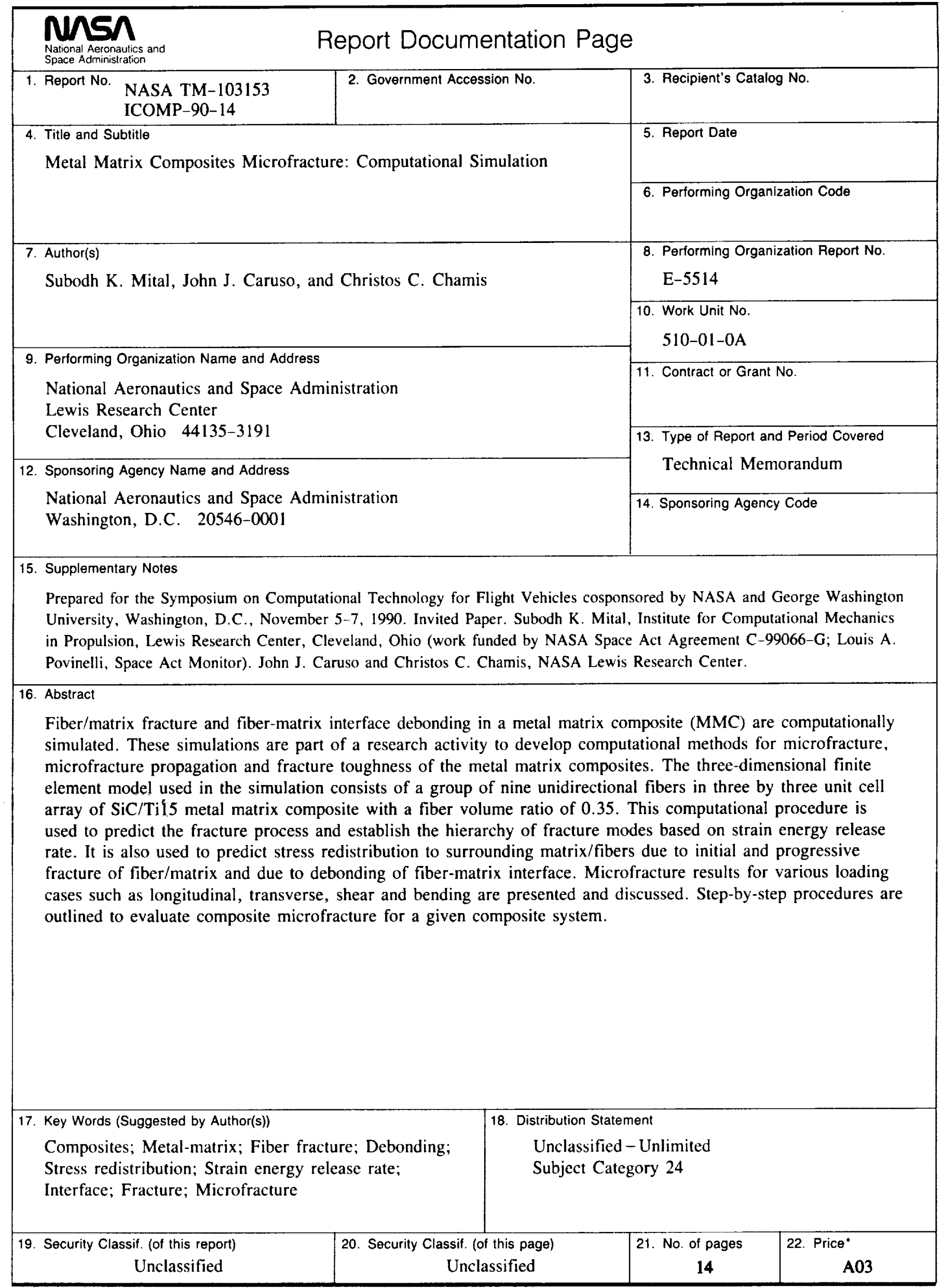




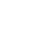

